This item was submitted to Loughborough's Research Repository by the author.

Items in Figshare are protected by copyright, with all rights reserved, unless otherwise indicated.

\title{
Guidance and control in a Josephson charge qubit
}

PLEASE CITE THE PUBLISHED VERSION

http://dx.doi.org/10.1103/PhysRevB.70.214521

\section{PUBLISHER}

(C) American Physical Society

\section{VERSION}

VoR (Version of Record)

\section{LICENCE}

CC BY-NC-ND 4.0

\section{REPOSITORY RECORD}

Ralph, J.F., E.J. Griffith, T.D. Clark, and Mark J. Everitt. 2019. "Guidance and Control in a Josephson Charge Qubit”. figshare. https://hdl.handle.net/2134/12068. 
This item was submitted to Loughborough's Institutional Repository (https://dspace.lboro.ac.uk/) by the author and is made available under the following Creative Commons Licence conditions.

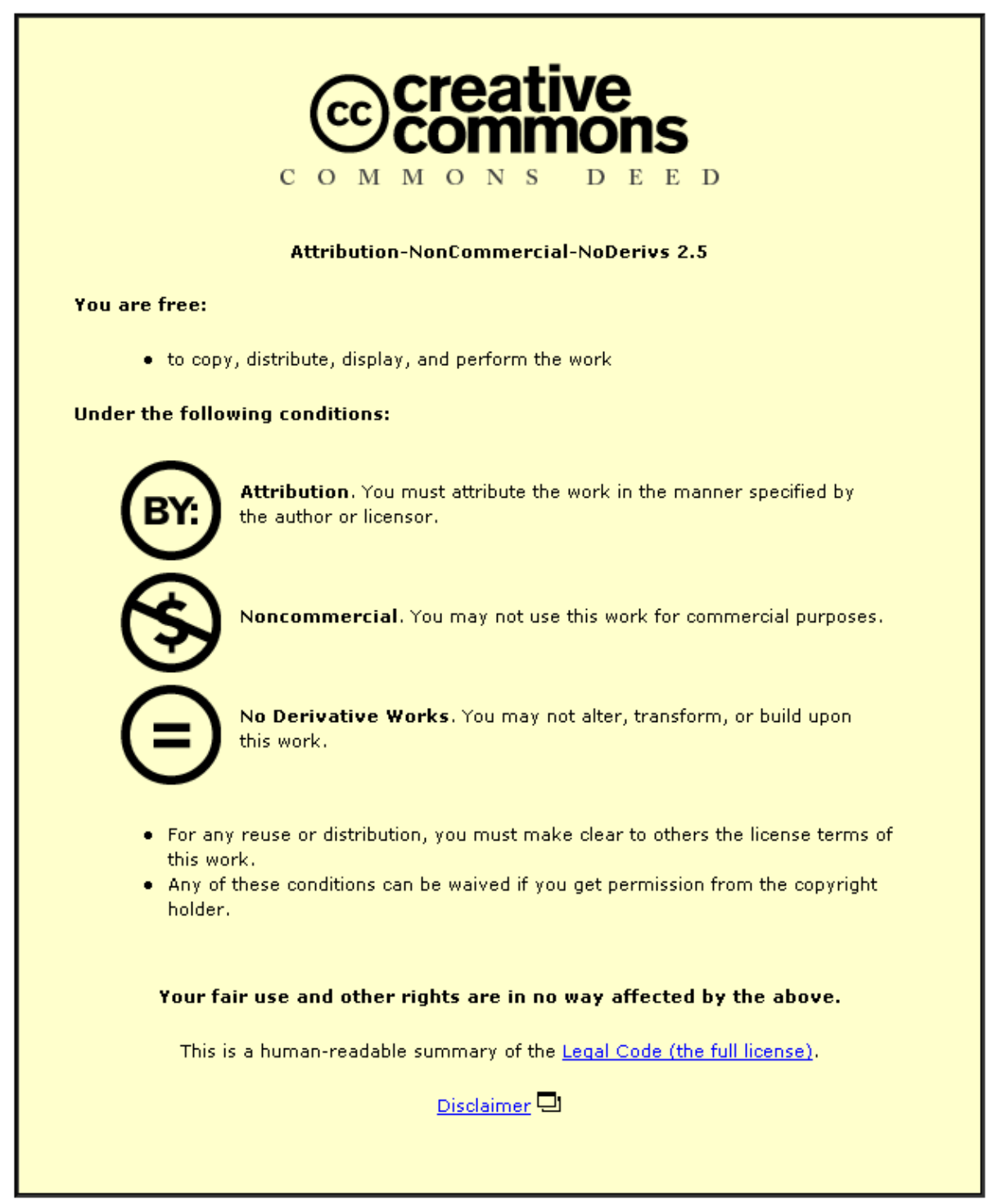

For the full text of this licence, please go to: http://creativecommons.org/licenses/by-nc-nd/2.5/ 


\title{
Guidance and control in a Josephson charge qubit
}

\author{
J. F. Ralph, ${ }^{1, *}$ E. J. Griffith, ${ }^{1}$ T. D. Clark, ${ }^{2}$ and M. J. Everitt ${ }^{2}$ \\ ${ }^{1}$ Department of Electrical Engineering and Electronics, The University of Liverpool, Brownlow Hill, \\ Liverpool, L69 3GJ, United Kingdom \\ ${ }^{2}$ School of Engineering, The University of Sussex, Falmer, Brighton BN1 9QT, United Kingdom
}

(Received 17 August 2004; published 23 December 2004)

\begin{abstract}
In this paper we propose a control strategy based on a classical guidance law and consider its use for an example system: a Josephson charge qubit. We demonstrate how the guidance law can be used to attain a desired qubit state using the standard qubit control fields.
\end{abstract}

DOI: 10.1103/PhysRevB.70.214521

PACS number(s): 74.50.+r, 03.65.-w, 85.25.Dq

\section{INTRODUCTION}

The practical operation of devices for quantum information processing is dependent upon the ability to control the behavior of the component qubits via external classical control fields. As in classical devices, the control/bias fields allow the operator to define the dynamical characteristics of the system. Bias fields contain noise that will couple into the device and can ultimately limit the coherent evolution of a quantum information processing system. In classical systems, a feedback control loop is often used to reduce the effect of such environmental noise or other unforseen perturbations on the evolution of the system. The control loop compares the desired behavior with the actual behavior of the system and aims to minimize the error between the two. The problem for quantum control is that a feedback loop requires some form of measurement to be made, and this measurement will often adversely affect the coherence of the quantum evolution. Several groups have suggested methods to overcome this problem, using techniques developed (mainly in quantum optics) to describe "weak" measurements. These measurements can be used to obtain information about a quantum system over a period of time while minimizing the adverse effects of the measurement interaction (see Ref. 1 for a recent review and a description of the relationship between classical control and quantum control). Closed-loop techniques fall into two main categories: Markovian feedback ${ }^{2-4}$ and Bayesian (or optimal) feedback. ${ }^{1,5,6}$ The first method uses the results of measurements to directly alter the external control fields applied to the system. The second builds an estimate of the system state over a number of measurements. Although they were developed in quantum optics, these techniques and related analysis have recently been applied to the control of solid-state qubits. ${ }^{7,8}$

This paper deals with an associated problem, that of generalizing the techniques of classical guidance (see, for example, Ref. 9) to the operation and manipulation of qubits. The main conceptual difference between guidance and control is one of timeliness. In control systems, the desired state of the system (classical or quantum) may be static or change with time, but there is always an error between the actual state of the system and the desired state. A control is applied to remove this error signal. In guidance systems, the evolution of the system is not as important as the final state. The controls are applied throughout the evolution to ensure that the system reaches the desired state at the desired time. In Ref. 10 Bouten et al. have addressed this problem implicitly, by using dynamical programming to solve an optimal control problem by minimizing the controls (which define a "cost" function) applied over the time available $\left(t_{\max }\right)$. Experience with classical guidance techniques shows that, while such algorithms may give a minimum cost solution, optimal control guidance can be difficult to implement and simpler guidance laws often provide sufficient accuracy with significantly simpler guidance-control systems. ${ }^{9}$ The most commonly used guidance law is referred to as proportional navigation, which is used in a wide variety of aerospace guidance systems (autopilots, guided missiles, etc.). Several variants of proportional navigation exist, but-in its most general form-it can be written as ${ }^{9}$

$$
a_{c}=N^{\prime} \frac{(\mathrm{ZEM})}{t_{g o}^{2}},
$$

where $a_{c}$ is the control (acceleration command) that should be applied to the system, $N^{\prime}$ is a constant (called the "navigation constant") which determines the strength of the commands, $t_{g o}$ is the time to go until the objective $\left(t_{\text {max }} \geqslant t_{g o}\right.$ $\geqslant 0$ ), and ZEM is the zero effort miss (that is, the distance between the desired state - the intercept point-and the predicted state if no more controls are applied). In classical guidance, an intercept is assured as long as $N^{\prime}>2$ and the accelerations commanded are achievable. In practice, $N^{\prime}$ is normally in the range $N^{\prime}=4 \rightarrow 6$, so that the controls immediately prior to intercept are minimized.

Proportional navigation guidance is not optimal in the sense of minimizing the controls applied, but is generally easier to implement in a practical control system and the controls that need to be applied to the system tend to have a lower bandwidth than those generated by more sophisticated algorithms. In this paper, we will generalize this classical guidance law to the problem of controlling a solid-state qubit. We will show some examples of the behavior predicted for an (open-loop) proportional guidance law applied to a Josephson charge qubit (e.g., Refs. 11 and 12). (Open-loop control, i.e., without feedback, has been studied in the context of atomic and quantum optics but not in the same form as that presented here ${ }^{13}$ ). In particular, we consider the robustness of the guidance law to noise in the bias fields and 
(a)
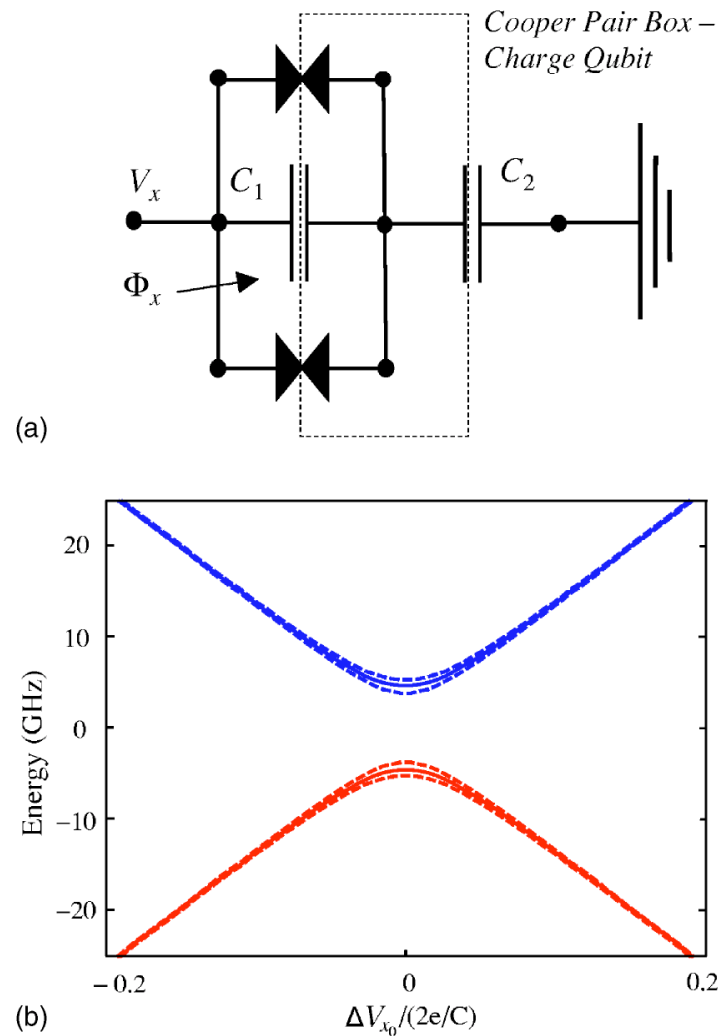

FIG. 1. (Color online) (a) Schematic of qubit circuit, showing capacitances $C_{1}+C_{2}=C$; and (b) energy levels for qubit as a function of $V_{x}$, using parameters given in the text and showing energies for nominal bias point $\Phi_{x_{0}}=0.25 \Phi_{0}$ (solid lines) and for extremes of the control modulation field $\Phi_{x_{0}}=(0.25 \pm 0.05) \Phi_{0}$ (dashed lines).

the introduction of a first-order time delay (low pass filter) into the control system, to investigate the effect of restricting the bandwidth of the control signal. Consideration is also given to the introduction of a simple measurement interaction and feedback control loop.

\section{JOSEPHSON CHARGE QUBIT}

The qubit studied in this paper is a commonly used (idealized) model for a standard experimental configuration. It consists of a superconducting island (a Cooper pair box) coupled to an external circuit via two parallel Josephson junctions $^{11,12}$ (see Fig. 1). The qubit has two main control fields: a voltage bias $\left(V_{x}\right)$ to control the energy of the charge states and a magnetic flux $\left(\Phi_{x}\right)$ to control the tunneling of electron pairs between the box and the external circuit. The two parallel Josephson junctions form a current loop and applying a magnetic field through this loop acts to modulate the tunneling rate onto and off the Cooper pair box. (In this paper, we assume that the two Josephson junctions are identical for simplicity. In practice, there will be small variations in the tunneling rates for each junction in any experimental system and it might be necessary to characterize these differences in a real system.) The effect of modulating the effective tunneling frequency on the qubit energy levels is shown in Fig. 1(b). We shall use circuit parameter values based on the experimental values given in Refs. 11 and 12 to ensure that the circuit parameters are realizable. In most experiments that have been reported using such systems (as well as in other superconducting qubit experiments based on persistent current devices), ${ }^{15}$ excited states are generated in the qubit by applying an additional field, a time-dependent microwave drive field. In this paper, we do not use an additional (external) microwave drive which reduces the complexity of the control system. This point it discussed in more detail below.

The Hamiltonian for the (two-state) qubit can be written in the charge basis representation as ${ }^{11,12}$

$$
H_{0}=\left(\begin{array}{cc}
\frac{C V_{x}^{2}}{2} & -\frac{\hbar \nu}{2} \cos \left(\frac{\pi \Phi_{x}}{\Phi_{0}}\right) \\
-\frac{\hbar \nu}{2} \cos \left(\frac{\pi \Phi_{x}}{\Phi_{0}}\right) & \frac{\left(2 e-C V_{x}\right)^{2}}{2 C}
\end{array}\right),
$$

where the basis states are zero excess pairs $(|0\rangle)$ and one excess pair $(|1\rangle)$ on the Cooper pair box, $C$ is the net capacitance of the island/box (in this case, we take $C=6 \times 10^{-16} \mathrm{~F}$ ), and $\nu$ is the tunneling (angular) frequency of the Josephson junction. The maximum Josephson tunneling frequency is taken to be $\nu / 2 \pi \simeq 12.9 \mathrm{GHz}$, although the effective Josephson frequency at the nominal bias point $\Phi_{x}=\Phi_{0} / 4$ is approximately $9.1 \mathrm{GHz}$ (see below), in line with the parameters given in Ref. 12 , where $\Phi_{0}=h / 2 e=2 \times 10^{-15} \mathrm{~Wb}$ is the superconducting flux quantum. For the purposes of this paper, we take the charge basis to be the computational basis for the qubit. The energy eigenstates are functions of the bias fields. Although we we will consider transitions between energy eigenstates under the action of the guidance/control, this is not necessary. As discussed below, we use the ground state as the initial state for convenience, since it is assumed that the system will relax to this state after some period of time, under the action of whatever dissipation processes are present in the system.

This Hamiltonian (or any other $2 \times 2$ Hermitian matrix) may be decomposed into four components, which correspond to a constant multiplied by the identity matrix $(I)$ or one of the three Pauli matrices

$$
\begin{gathered}
\sigma_{x}=\left(\begin{array}{ll}
0 & 1 \\
1 & 0
\end{array}\right), \quad \sigma_{y}=\left(\begin{array}{cc}
0 & -i \\
i & 0
\end{array}\right), \\
\sigma_{z}=\left(\begin{array}{cc}
1 & 0 \\
0 & -1
\end{array}\right)
\end{gathered}
$$

We write the bias voltage as $V_{x}=e / C+\Delta V_{x}$, and then decompose the Hamiltonian as

$$
\begin{aligned}
H_{0} & =H_{I} I+H_{x} \sigma_{x}+H_{y} \sigma_{y}+H_{z} \sigma_{z} \\
& =\left(\frac{C\left(\Delta V_{x}\right)^{2}}{2}+\frac{e^{2}}{2 C}\right) I+e\left(\Delta V_{x}\right) \sigma_{z}-\frac{\hbar \nu}{2} \cos \left(\frac{\pi \Phi_{x}}{\Phi_{0}}\right) \sigma_{x} .
\end{aligned}
$$

From this decomposition, it is easy see that there is no $\sigma_{y}$ term in the basic Hamiltonian. This would be the term that would normally be responsible for exciting the qubit into the 
excited state and has been the most common control coupling to be studied in quantum optics. ${ }^{4,6,10,16}$ To generate a $\sigma_{y}$ term, it is necessary to apply a time-dependent field, since $\sigma_{x}$ and $\sigma_{z}$ do not commute: $\left[\sigma_{z}, \sigma_{x}\right]=2 i \sigma_{y}$. Normally, in quantum optics, a laser is used to pump a qubit into an excited state, or in solid-state experiments (such as those described in Refs. 11 and 12) an external microwave source is used. In an experimental system, the underlying Hamiltonian may not be exactly what is predicted by the idealized model used here, but a number of techniques have been proposed to allow the deviations to be characterized. ${ }^{14}$ The use of an external microwave source in solid state is not ideal for largescale systems because of potential problems in isolating qubits from drives applied to neighboring devices. Because of this, we restrict ourselves to controls that arise from timedependent bias fields $\Delta V_{x}$ and $\Phi_{x}$ and consider the effect of limiting the bandwidth of these fields in a later section.

The general representation for a qubit state is

$$
|\psi\rangle=\cos \left(\frac{\theta}{2}\right)|0\rangle+\sin \left(\frac{\theta}{2}\right) e^{i \phi}|1\rangle
$$

where $\theta \in[0, \pi]$ and $\phi \in[0,2 \pi]$, which can be written as a (pure state) density matrix $\rho=|\psi\rangle\langle\psi|$

$$
\rho=\left(\begin{array}{cc}
\cos ^{2}\left(\frac{\theta}{2}\right) & \cos \left(\frac{\theta}{2}\right) \sin \left(\frac{\theta}{2}\right) e^{-i \phi} \\
\cos \left(\frac{\theta}{2}\right) \sin \left(\frac{\theta}{2}\right) e^{i \phi} & \sin ^{2}\left(\frac{\theta}{2}\right)
\end{array}\right) .
$$

However, the most convenient representation for the purposes of this paper is the Bloch sphere representation, ${ }^{17}$ where the two angles $\theta$ and $\phi$ represent angles on a unit sphere, defined in a three-dimensional space by

$$
\left(\begin{array}{c}
X \\
Y \\
Z
\end{array}\right)=\left(\begin{array}{c}
\sin \theta \cos \phi \\
\sin \theta \sin \phi \\
\cos \theta
\end{array}\right)=\left(\begin{array}{c}
\rho_{01}+\rho_{10} \\
i \rho_{01}-i \rho_{10} \\
\rho_{00}-\rho_{11}
\end{array}\right) .
$$

The different components $\left(\sigma_{x}, \sigma_{y}\right.$, and $\left.\sigma_{z}\right)$ present in the Hamiltonian represent rotations in this three-dimensional Bloch space (about the $X, Y$, and $Z$ axes, respectively). The fact that there is no $\sigma_{y}$ term in the basic Hamiltonian is not a problem, because it is possible to reach any point on the Bloch sphere from any other by successive rotations about any two (nonparallel) axes. The guidance algorithm simply governs the size of the rotations that are to be applied to achieve the objective.

\section{PROPORTIONAL GUIDANCE}

The classical proportional navigation algorithm predicts the expected miss distance if no control is applied [the zero effort miss ZEM], a quantum analog for the qubit can be developed in a similar manner. The evolution in the absence of controls is described by the basic Hamiltonian given in Eq. (2) and the time evolution of the wave function and (pure state) density matrix is governed by a unitary evolution operator

$$
\hat{U}(t)=\exp \left(-\frac{i H_{0} t}{\hbar}\right)
$$

The main difference between proportional navigation on a sphere and proportional navigation in three Euclidean dimensions is that the rotations generated by this unitary matrix and the rotations required to move the estimated final state onto the desired state do not commute. The rotation required at the end point will not produce the same effect if it is applied earlier in the trajectory. Because of this, we need to retrodict where the desired state should have been at the earlier time, ${ }^{18}$ if it is to end up at the desired state under the free evolution given by $H_{0}$. The ZEM in this case is the two angles ( $\theta_{Z E M}$ and $\left.\phi_{Z E M}\right)$ which separate the current estimated state (on the Bloch sphere) from the point where the desired state would have to be at the current time. So, we calculate

$$
\rho_{d}\left(t_{g o}\right)=\hat{U}^{\dagger}\left(t_{g o}\right) \rho_{d} \hat{U}\left(t_{g o}\right)=\exp \left(\frac{i H_{0} t_{g o}}{\hbar}\right) \rho_{d} \exp \left(-\frac{i H_{0} t_{g o}}{\hbar}\right),
$$

where $\rho_{d}$ is the desired final state (or "target state"). From this density matrix, we can calculate the two angles $\theta_{d}\left(t_{g o}\right)$ and $\phi_{d}\left(t_{g o}\right)$ which define the retrodicted state. The ZEM angles are then given by

$$
\begin{gathered}
\theta_{Z E M}=\theta_{d}\left(t_{g o}\right)-\theta, \\
\phi_{Z E M}=\phi_{d}\left(t_{g o}\right)-\phi,
\end{gathered}
$$

where $\theta$ and $\phi$ represent the current state (allowing for the periodicity of the angles). The controls that need to be applied are

$$
\begin{gathered}
\frac{d \theta_{c}}{d t}=N^{\prime} \frac{\left(\theta_{Z E M}\right)}{t_{g o}}, \\
\frac{d \phi_{c}}{d t}=N^{\prime} \frac{\left(\phi_{Z E M}\right) \sin \theta}{t_{g o}},
\end{gathered}
$$

where the controls are angular velocities rather than accelerations because the Bloch equations are first-order differential equations,${ }^{17}$ rather than second-order classical dynamics, and the $\sin \theta$ term arises because the differences in $\phi_{Z E M}$ near the poles of the Bloch sphere need to account for the curvature of the sphere.

The controls can be equated to an equivalent Hamiltonian by integrating over a small time interval $(\delta t)$ and using the fact that a $\sigma_{x}$ Hamiltonian generates rotations about the $X$ axis and a $\sigma_{z}$ Hamiltonian generates rotations about the $Z$ axis. We find the Hamiltonian that rotates the Bloch vector from its current position (as given by $\theta$ and $\phi$ ) through angles

$$
\delta \theta_{c}=N^{\prime}(\delta t) \frac{\left(\theta_{Z E M}\right)}{t_{g o}}
$$




$$
\delta \phi_{c}=N^{\prime}(\delta t) \frac{\left(\phi_{Z E M}\right) \sin \theta}{t_{g o}} .
$$

Making a linear approximation and solving for the Hamiltonian controls $\left(H_{x_{c}}\right.$ and $\left.H_{z_{c}}\right)$, we obtain expressions

$$
\begin{gathered}
H_{x_{c}}=-\frac{\hbar\left(\delta \theta_{c}\right)}{2(\delta t) \sin \phi}, \\
H_{z_{c}}=-\frac{\hbar\left(\delta \phi_{c}\right)}{2(\delta t)} .
\end{gathered}
$$

(In practice, although the expression for $H_{x_{c}}$ includes a $1 / \sin \phi$ term, removing the dependence upon $\phi$ does not affect the performance of the guidance to a large degree and it dramatically reduces the bandwidth required for the control signal and makes the control system more resilient to time delays.)

Applying these controls requires manipulating the bias fields $\Delta V_{x}$ and $\Phi_{x}$. Clearly there are limits to the size of the controls that can be applied using these bias fields. The two state approximation for the charge qubit is only valid as long as changes in the bias voltage are small $\left|\Delta V_{x}\right| \ll 2 e / C$, so we impose constraints such that $\left|\Delta V_{x_{c}}\right|<0.1 \times 2 e / C$, where $\Delta V_{x_{0}}$ is the nominal voltage bias point and the voltage bias control fluctuates about this point $\Delta V_{x}=\Delta V_{x_{0}}+\Delta V_{x_{c}}$. Here the capacitance that is used to apply the gate voltage, which in turn controls the voltage applied across the qubit, is assumed to be the same as the qubit island capacitance. This is not necessary, changing the gate capacitance simply rescales the voltage bias and the associated behavior under the action of the guidance/control. As long as this capacitance is known, the appropriate controls can be applied. For the magnetic flux bias, we must ensure that any fluctuation around the nominal bias point $\Phi_{x_{0}}$ is small enough so that the response is approximately linear. So $\Phi_{x}=\Phi_{x_{0}}+\Delta \Phi_{x_{c}}$, where $\Phi_{x_{0}}$ $=0.25 \Phi_{0}$ and $\left|\Delta \Phi_{x_{c}}\right|<0.05 \Phi_{0}$. Within this region, the cosine tunneling term is approximately linear in $\Delta \Phi_{x_{c}}$ and we obtain the following relations for the control fields:

$$
\begin{gathered}
\Delta \Phi_{x_{c}}=\frac{2 H_{x_{c}}}{\pi \hbar \nu \sin \left(\frac{\pi \Phi_{x_{0}}}{\Phi_{0}}\right)}, \\
\Delta V_{x_{c}}=\frac{H_{z_{c}}}{e} .
\end{gathered}
$$

Beyond the limits given, the control is assumed to have saturated and any controls commanded are unachievable. This limits the number of states that are reachable from a given initial state, but as long as the desired state falls within the reachable set for the initial state, this should not be a major problem. For simplicity, we assume that the initial state corresponds to the ground state for the unperturbed Hamiltonian $H_{0}$ at the nominal bias point ( $\Delta V_{x_{0}}$ and $\left.\Phi_{x_{0}}=0.25 \Phi_{0}\right)$. That is, for simplicity, we assume that the qubit has relaxed into the ground state via some (weak) dissipative process. Although it would be possible to prepare a different initial state by another process, such as some type of projective measurement interaction, this is not considered here.

The guidance algorithm operates by integrating the evolution of the qubit for a small timestep and applying controls via $\Delta V_{x}$ and $\Phi_{x}$ that are determined by finding the ZEM angles from Eqs. (6) and (7), converting these angles into an effective Hamiltonian using Eqs. (9) and (10), and finally converting these Hamiltonian controls to bias values via Eq. (11). Applying this procedure iteratively generates a timedependent bias signal that can then be applied to a qubit and should provide the desired state at the desired time $\left(t_{g o}=0\right)$ as long as it is within the reachable set. Of course, since the control is currently open loop, the actual state of the system is unknown. The controls are generated from the knowledge of where the state should be if it started in the ground state and the controls had been correctly applied. A wide variety of states may be prepared in this way and the controls have several distinct advantages: they have a comparatively low bandwidth, they operate via the standard bias fields without an additional external drive, and (for states within the reachable set) the controls vanish as $t_{g o} \rightarrow 0$. This last point means that the Hamiltonian is only weakly perturbed at the time when the state is required. Although the algorithm is presented as an open-loop control technique, it provides a natural generalization to feedback (closed-loop) control, which is discussed in a later section.

The difference between the desired state and the final (possibly mixed) state can be quantified in a variety of standard ways. For the purposes of this paper, we use three common measures to characterize the performance of the guidance algorithm. The first measure is the fidelity of the state, introduced by Jozsa, ${ }^{19}$ which is one measure for how close the final state is from the desired state. The fidelity $F$ for two density matrices $\rho_{f}$ and $\rho_{d}$ is given by ${ }^{20}$

$$
F=F\left(\rho_{f}, \rho_{d}\right)=\left|\operatorname{Tr}\left[\sqrt{\sqrt{\rho_{d}} \rho_{f} \sqrt{\rho_{d}}}\right]\right|^{2},
$$

which varies between zero and one (one being that the final state matches the desired state exactly). The second measure that we use in this paper is the trace distance $D^{21}$

$$
D=D\left(\rho_{f}, \rho_{d}\right)=\frac{1}{2} \operatorname{Tr}\left[\rho_{f}-\rho_{d}\right],
$$

which also runs from zero to one (zero being identical states) and measures the separation of the desired and final states. The final measure is the distance from the surface of the Bloch sphere, which is a measure of the purity (or, conversely, the mixedness) of the state, and can be written as ${ }^{4,16}$

$$
p=2 \operatorname{Tr}\left[\rho^{2}\right]-1,
$$

with pure states giving $p=1$ and maximally mixed states giving $p=0$. The purity is most important for mixed states, which are generated by the inclusion of stochastic terms such as the measurement and feedback model discussed in a later section.

\section{IDEAL OPEN-LOOP PERFORMANCE}

Starting the qubit in its ground state, we can pick a particular target state and calculate the control signal required to 

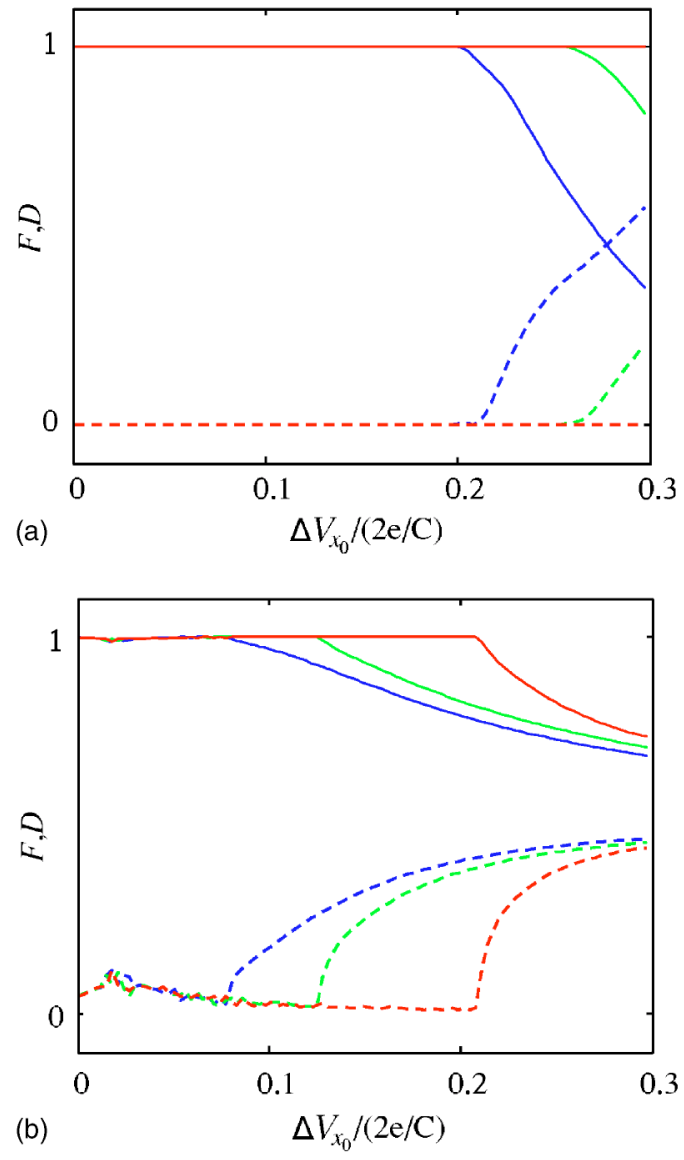

FIG. 2. (Color online) Fidelity ( $F$, solid lines) and trace distance $(D$, dashed lines) achieved using guidance algorithm as a function of the voltage bias value for different values of $t_{\max }$ : 20 qubit cycles (blue), 50 qubit cycles (green), and 100 qubit cycles (red); (a) target state is the excited state at nominal bias voltage, and (b) target state is an arbitrary state with $\theta=\pi / 2$ and $\phi=\pi / 4$.

generate this state at a later time using the technique described above. We start by examining the performance of the guidance algorithm when applied to an ideal system (with no errors) and then examine the performance in the presence of possible experimental errors.

Although any target state can be chosen, an obvious candidate is the qubit excited state (which is a function of the nominal bias fields). As we have said, we assume that the initial qubit state will be the ground state, the qubit having relaxed into this state prior to the control being applied. Figure 2(a) shows the fidelity and trace distance achieved for a range of $\Delta V_{x_{0}}$ and for three different time periods $t_{\max }=20,50,100$ qubit cycles and $N^{\prime}=8$. The purity is trivially one, since there is no stochastic evolution and the qubit state is therefore pure. We see from the graphs that the excited state is easier to reach (high fidelity and low trace distance) from voltage values close to zero, $\Delta V_{x_{0}} \simeq 0$. The fidelity is one for a wide range of bias voltages, even when the time available is relatively short, $t_{\max }=20$ cycles. The main reason that the excited state is easier to reach near zero bias is that the ground and excited states at zero bias are equal (symmetric and antisymmetric) superpositions of the two basis states, and the main controls that are required are rota- tions about the $Z$ axis $\left(\sigma_{z}\right)$, which are easier to achieve than rotations about the $X$ or $Y$ axes. As the bias voltage is increased, the ground and excited states shift toward the poles of the Bloch sphere (and the qubit natural oscillation frequency increases, reducing the time available), and more controls are required from the magnetic bias field (which generates $\sigma_{x}$ terms). However, allowing more time for the guidance (by extending $t_{\text {max }}$ ) increases the ability to generate an excited state, and for $t_{\max }=100$ cycles the excited state is within the reachable set for the whole of the range $\Delta V_{x_{0}}$ shown in Fig. 2(a). Selecting the excited state as a target state has one more practical advantage. Because the exited state is stationary by definition, the production of an excited state is quite robust. Slight variations in the control fields or the time to go only generate small deviations from the excited state.

Figure 2(b) shows the performance of the guidance algorithm for a target state that is not an energy eigenstate for any values of the bias fields. We select an equal superposition $(\theta=\pi / 2)$ but with a phase $\phi=\pi / 4$. Here we see that the reachable set (in terms of $\Delta V_{x_{0}}$ ) is very much reduced compared to the previous example. Even though the fidelity is near one for a large range of bias voltages, the trace distance is significantly greater than zero until $t_{\max } \simeq 100$ cycles and $\Delta V_{x_{0}} \simeq 0.2 \times 2 e / C$. This is a result of the fact that the target state is not an energy eigenstate and is therefore nonstationary for all bias values. The control is therefore much more sensitive to small variations.

Figure 3 shows an example of the evolution of the qubit state under the proportional navigation guidance and the control fields that were applied. The evolution starts in the ground state and rapidly spirals around the Bloch sphere under the influence of the control, and gradually approaches the excited state, spiraling in gradually as the controls applied reduce in size. This is a good example of the benefit of this approach, where the controls subside to zero as the system approaches the desired state. This has distinct experimental advantages because the bias fields will be static immediately prior to $t_{g o}=0$, which means that the Hamiltonian is not varying rapidly when the desired state is required. It is also noticeable that both control fields contain a dominant frequency component that matches the coherent oscillation frequency of the qubit, indicating that a coherent drive at the transition frequency is an important part of the control fields.

\section{IMPERFECT BIAS FIELDS}

In classical guidance systems the guidance-control feedback loop must be robust enough so that small perturbations from noise or imperfections in the control system are damped out and the system achieves its objective. The main imperfections in classical guidance tend to come from uncertainties in the physical parameters that define the transfer function between the accelerations commanded by the guidance law and the actual accelerations achieved by the controls. In the qubit guidance case, this is more difficult because of the problems already mentioned in measuring the error signal and the algorithm discussed so far is an openloop control system. Instead, we require that the fidelity and 


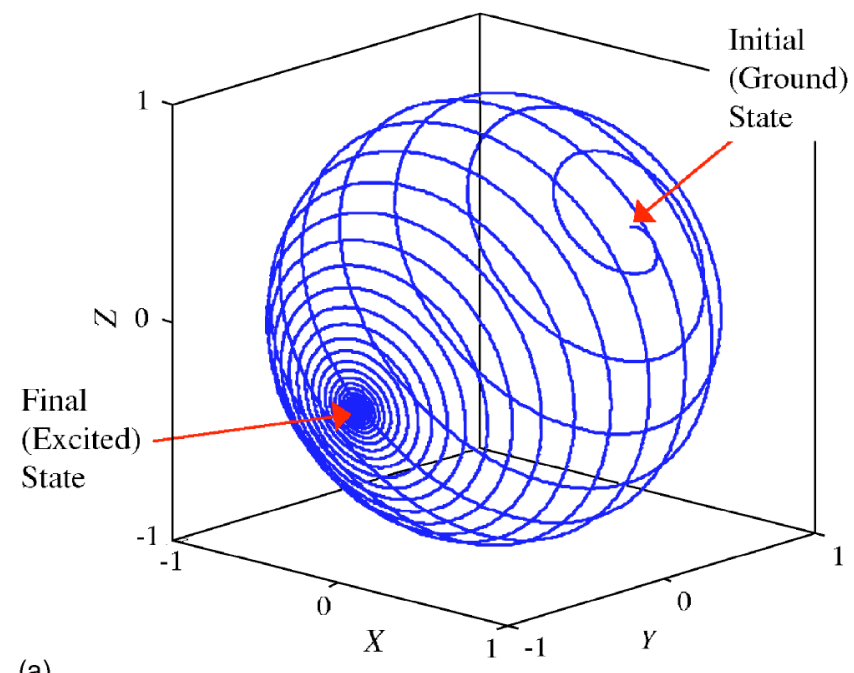

(a)

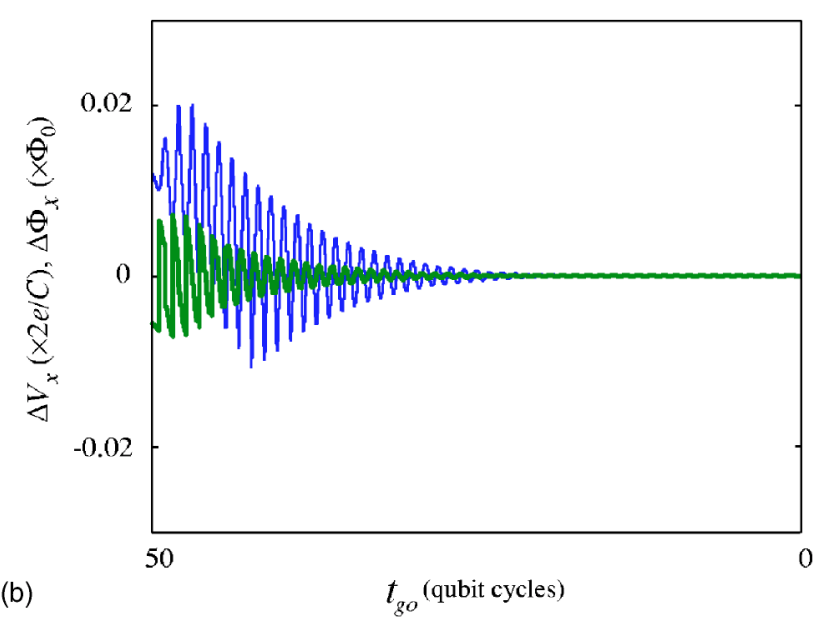

FIG. 3. (Color online) (a) Example trajectory of state on the Bloch sphere under proportional guidance with the excited state as the target state and $\Delta V_{x_{0}}=0.1 \times 2 e / C$; and (b) control fields $\Delta V_{x}$ (green) and $\Delta \Phi_{x_{c}}$ (blue) as a function of the $t_{g o}$, for example, trajectory in (a).

trace distance of the final state be weakly sensitive to small variations in the experimental control parameters. For example, the static (nominal) bias point may only be known to a certain accuracy. We should require that the final state approximately correct even if the static bias field is slightly off or if a small amount of dynamical noise is present in the fields. This is also of concern when the qubit is nonideal, either the gate capacitance is only known to a finite accuracy (so the scaling of the voltage biases is inaccurate) or the Hamiltonian includes nonideal terms (possibly due to variations in the junctions coupling the island to the bulk material). In these cases the controls applied will not necessarily generate rotations about axes exactly aligned to the the $X$ and $Z$ axes.

In practice, we find that-in common with the performance of the guidance algorithm itself-the sensitivity to noise is dependent on the target state. For static errors of the order of $\Delta\left(\Delta V_{x_{0}}\right) \simeq 10^{-5} \times 2 e / C$ and $\Delta\left(\Phi_{x_{0}}\right) \simeq 10^{-4} \Phi_{0}$, the performance of the algorithm for the example given in Fig.

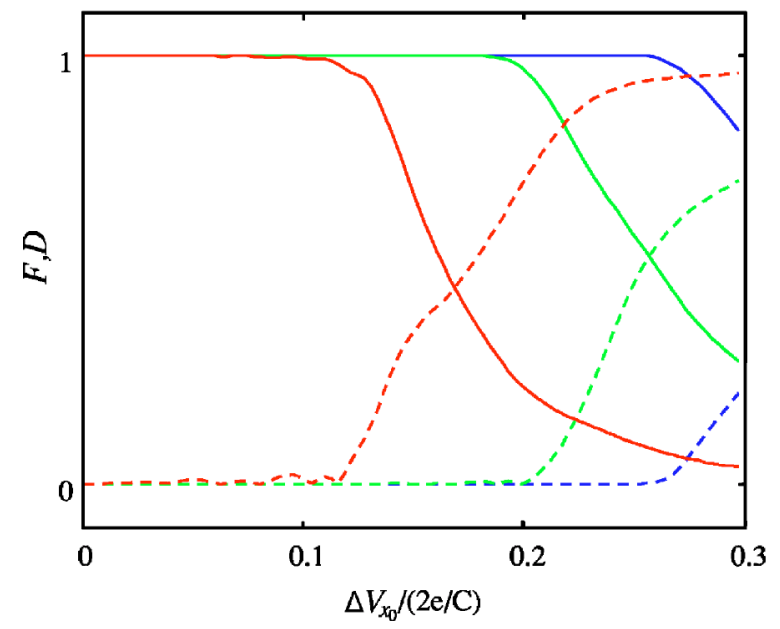

FIG. 4. (Color online) Fidelity ( $F$, solid lines) and trace distance $(D$, dashed lines) achieved using guidance algorithm as a function of the voltage bias value for different time delays: zero time delay (blue), 0.125 qubit cycle delay (green), and 0.25 qubit cycle delay (red); target state is the excited state at nominal bias voltage and $t_{\text {max }}=50$ cycles.

2(a) (i.e., the target state is the excited state) is very good. (The performance is also good for time-dependent errors, as long as the cumulative errors during the control cycle are of the same order as these tolerances.) Even for variations 1 order of magnitude larger than this, the performance is still acceptable for the excited state over a comparatively wide range of bias voltages, even if the reachable set is significantly reduced. The accuracy of the final state for the target state given in Fig. 2(b) would be significantly less with errors of this size. The trace distance between the final state and desired state is noticeably larger when bias errors are introduced. For dynamical noise, we have also characterized the performance of the algorithm in the presence of white noise (i.e., uncorrelated with a uniform frequency distribution), and the performance is similar to that for static bias errors as long as the cumulative drift of the bias fields is less than the limits given above.

\section{FIRST-ORDER TIME DELAYS}

In addition to noise, an experimental system is also likely to contain other imperfections. The main one considered here is a constraint on the bandwidth allowed for the control signal. We use a simple method of restricting the bandwidth in the control system by introducing a first-order time delay, which acts as a low-pass filter and has a transfer function (Laplace transform) given by ${ }^{22}$

$$
F(s)=\frac{1}{1+s T_{d}},
$$

where $T_{d}$ is the time delay constant. An example of this type of delay is a low-pass $R C$ filter, which should be familiar from standard electrical circuit analysis. The effect of this filter is to exponentially damp rapid variations in the controls, and in Fig. 4 we show the effect of such time delays on 
the performance of the guidance algorithm. Time delays on the order of $T_{d} \simeq 0.1-0.2$ cycles have little effect on the fidelity or trace distance of the final state for bias voltages close to zero, but toward the far right (higher voltage biases), the excited state becomes harder to reach, indicating that the bandwidth of the control signal is larger at these extreme values. By contrast, the effect of first-order time delays on the case shown in Fig. 2(b) would actually be less pronounced than that shown in Fig. 4 because the range of bias values from which the target state is reachable is already comparatively small.

\section{CLOSED-LOOP PERFORMANCE}

In this section we consider the use of a simple measurement and Markovian feedback mechanism to demonstrate how the open-loop guidance approach could be adapted using existing quantum feedback techniques. As an example, we choose a simple model for photon detection and (instantaneous) feedback. The model assumes that the qubit is weakly coupled to a lossy reservoir and that projective measurements are made on this reservoir. The results of the measurement are then used to modify the controls applied to the qubit. This model may not be entirely realistic, because of problems with detecting single microwave photons and with the large bandwidths required for a rapid feedback, but it demonstrates the general approach. The basic idea is to apply the guidance algorithm as described above and to modify the control pulses, to allow for the reduced time to go, when a photon is emitted and detected (detection is assumed to occur with efficiency $\eta$ ). Where photons are not detected, the qubit will still be coupled to the lossy reservoir which will cause an additional (nonunitary) perturbation on the otherwise coherent dynamics of the system and this is allowed for in the modeling but not in the guidance-control algorithm.

The measurement mechanism is modeled using a quantum trajectory approach, ${ }^{23,24}$ corresponding to an unraveling of the Markovian Master equation for the qubit reduced density operator (after performing a partial trace over the lossy reservoir). In this paper, we choose the quantum jumps approach, ${ }^{23,24}$ which is suitable for modeling spontaneous emission processes and is computationally efficient. ${ }^{25}$ Physically, this unraveling corresponds to the detection or absorption of spontaneously emitted photons on a time scale that is significantly faster than any of the time scales present in the quantum system. All unravelings reproduce the Master equation evolution when averaged, and the individual quantum "trajectories" for the qubit are described by a model given in Ref. 25 .

The spontaneous emission process and subsequent detection of the photon introduces quantum jumps that project into the instantaneous ground state of the qubit. The probability of a spontaneous decay occurring during a small-but finite-time interval $\delta t$ is $\gamma\left\langle\hat{c}^{\dagger} \hat{c}\right\rangle \delta t$, where $\hat{c}^{\dagger}$ and $\hat{c}$ are the raising and lowering operators for the (instantaneous) qubit energy states, respectively. During each time interval where no spontaneous decay occurs, a nonunitary evolution operator
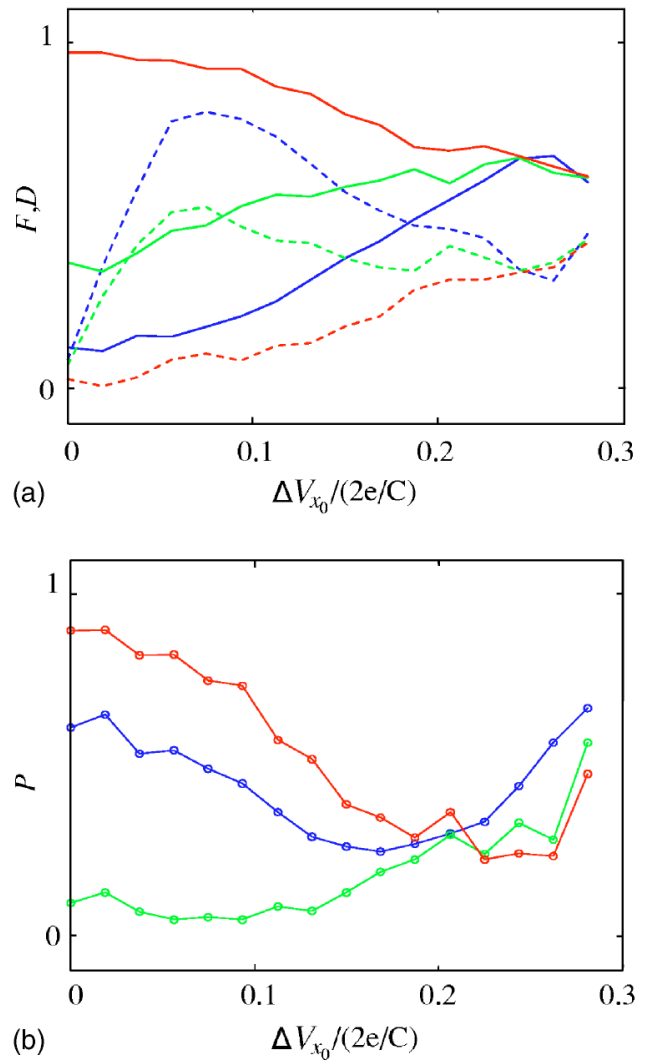

FIG. 5. (Color online) (a) Fidelity ( $F$, solid lines) and trace distance ( $D$, dashed lines), and (b) purity ( $p$, circles) achieved using guidance algorithm as a function of the voltage bias value for $\gamma$ $=0.05 /$ cycle and $\eta=0.0$ (no feedback, blue), $\eta=0.5$ (green), and $\eta=1.0$ (red); target state is the excited state at nominal bias voltage and $t_{\text {max }}=50$ cycles.

$$
\hat{\Omega}_{0}(d t)=1-\frac{i}{\hbar} \hat{H}_{q u} \delta t-\frac{\gamma}{2} \hat{c}^{\dagger} \hat{c} \delta t
$$

is applied to the qubit state. When a decay occurs, an operator

$$
\hat{\Omega}_{1}(d t)=\sqrt{\gamma \delta t} \hat{c}
$$

is applied to the qubit state. Each run of the simulation produces a subjective trajectory. For each trajectory, feedback control is invoked if a photon is detected, with probability $\eta$, so that the state evolution is conditional upon the detections and then averaged over multiple realizations. The result is averaged over many runs to provide an estimate of the mixed state density matrix for the qubit, from which we can calculate purity, fidelity, and trace distance. [Some noise is still present in the average density matrix and the performance measures, but this is relatively small and is due to the limited number of runs (typically 500-1000) which is dictated by computational constraints.]

Figure 5 shows an example of the fidelity, trace distance, and purity for this simple closed-loop control system, corresponding to one of the examples shown in Fig. 2(a) with the damping rate $\gamma=0.05 /$ qubit cycle. If the damping rate were significantly smaller than this, the probability of a qubit un- 
dergoing a spontaneous decay during the period of control would be negligible and the system would reduce to the open-loop case already discussed. If the damping rate were significantly higher than this, guidance control (open loop or closed loop) would be impossible. For the case shown, the probability of undergoing a transition during the control period is significant. Without feedback (or very inefficient photon detection $\eta=0$ - blue lines) the fidelity and trace distance are very low for small voltage bias values, and the purity is fairly high. This is an indication that most of the qubits will spontaneously emit photons and decay back to the ground state. The main controls are applied near the start of the control period [see Fig. 3(b)], which will tend to leave the qubit near the ground state once a photon has been emitted. In fact, the two properties are related, since the qubit is only likely to emit a photon once the control has brought the system close to the excited state in the first place. As the bias voltages are increased, the excited state is harder to reach (and therefore occurs later in the control cycle) and the emission probability consequently goes down. This is the cause of the minimum in the purity for the $\eta=0$ case, where the two effects balance out, so that the mid-range voltages are more likely to be mixed between the ground and excited states.

Where the detection probability is nonzero (the green and red curves in Fig. 5), feedback is allowed and the controls can be modified when a photon is detected. In the case of $\eta=0.5$ the purity is significantly reduced because there is a chance of being near either state for most values of the bias voltage, either through decay and detection, decay and nondetection, and nondecay. However, as desired, the fidelity increases as the detection probability increases, indicating that the feedback is working correctly. Even so, even with $\eta=1$, the closed loop performance does not reach the openloop, nondissipative performance. This is because the spontaneous emission reduces the effective time available for the control, and multiple jumps are likely to occur for some bias voltages. The average number of jumps is dependent on $\eta$ and $\Delta V_{x_{0}}$, but for $\eta=1$ and $\Delta V_{x_{0}} \simeq 0$, two or more jumps are not uncommon.

\section{DISCUSSION}

In this paper we have presented a generalization of a classical guidance law to the problem of control of a qubit state on the Bloch sphere. We have chosen the proportional navigation guidance law because of its relative simplicity and its resultant widespread use in classical guidance and control systems. We have demonstrated that this guidance law can be used to generate an arbitrary quantum state from the ground state of a superconducting charge qubit using the standard control fields (voltage and magnetic flux bias). The controls produced by this guidance law are relatively robust to imperfections in the control fields and to first-order time delays, implying that the control signals have a comparatively low bandwidth. This should make it possible to control the state of the qubit using signals fed through low-pass transmission lines. We have also suggested a simple method to allow the generalized guidance law to be included in a closed-loop (Markovian) control system.

Although the ability to control the state of a single qubit with a high degree of accuracy is important for a possible quantum information processing device, the ability to control the collective behavior of multiple qubits is also of great interest. The ability to visualize the control of the qubit state on the Bloch sphere is useful in understanding the guidance mechanism, but it is not essential. Generalizing the guidance algorithm to higher-dimensional settings (multiple qubits or $N$-level systems) simply requires an understanding of the group structure of the space and the ability to create appropriate control Hamiltonians from the generators of the group. ${ }^{26}$ As long as the group generators (or the restricted set of generators available to the control system) allow the state space to be explored fully, then it should be possible to generalize the guidance algorithm described in this paper to higher-dimensional systems.
*Electronic address: jfralph@liv.ac.uk

${ }^{1}$ A. C. Doherty, S. Habib, K. Jacobs, H. Mibuchi, and S. M. Tan, Phys. Rev. A 62, 012105 (2000).

${ }^{2}$ H. M. Wiseman and G. J. Milburn, Phys. Rev. A 49, 1350 (1994).

${ }^{3}$ H. F. Hofmann, G. Mahler, and O. Hess, Phys. Rev. A 57, 4877 (1998).

${ }^{4}$ J. Wang and H. M. Wiseman, Phys. Rev. A 64, 063810 (2001).

${ }^{5}$ V. P. Belavkin, Rep. Math. Phys. 45, 353 (1999).

${ }^{6}$ A. C. Doherty and K. Jacobs, Phys. Rev. A 60, 2700 (1999).

${ }^{7}$ A. N. Korotkov, Phys. Rev. B 60, 5737 (1999); R. Ruskov and A. N. Korotkov, ibid. 66, 041401 (2002); A. N. Korotkov, quantph/0404696 (unpublished).

${ }^{8}$ N. P. Oxtoby, P. Warszawski, H. M. Wiseman, R. E.S. Polkinghorne, and H.-B. Sun, quant-ph/0401204 (unpublished).

${ }^{9}$ P. Zarchan, Tactical and Strategic Missile Guidance, 3rd ed., Progress in Astronatics and Aeronautics Vol. 176 (AIAA Press, Reston, VA, 1997).
${ }^{10}$ L. Bouten, S. Edwards, and V. P. Belavkin, quant-ph/0407192 (unpublished).

${ }^{11}$ Y. Nakamura, Yu. A. Pashkin, and J. S. Tsai, Nature (London) 398, 786 (1999).

${ }^{12}$ Yu. A. Pashkin, T. Yamamoto, O. Astaflev, Y. Nakamura, D. V. Averin, and J. S. Tsai, Nature (London) 421, 823 (2003).

${ }^{13}$ L. Viola, E. Knill, and S. Lloyd, Phys. Rev. Lett. 82, 2417 (1999); S. G. Schirmer, H. Fu, and A. I. Solomon, Phys. Rev. A 63, 063410 (2001).

${ }^{14}$ J. F. Ralph, T. D. Clark, M. J. Everitt, H. Prance, P. Stiffell, and R. J. Prance, Phys. Lett. A 317, 199 (2003); S. G. Schirmer, A. Kolli, and D. K. L. Oi, Phys. Rev. A 69, 050306 (2004).

${ }^{15}$ J. R. Friedman, V. Patel, W. Chen, S. K. Tolpygo, and J. E. Lukens, Nature (London) 406, 43 (2000); C. H. van der Wal, A. C.J. ter Haar, F. K. Wilhem, R. N. Schouten, C. J.P.M. Harmans, T. P. Orlando, S. Lloyd, and J. E. Mooij, Science 290, 773 (2000); J. M. Martinis et al., Phys. Rev. Lett. 89, 117901 (2002). 
${ }^{16}$ H. M. Wiseman, S. Mancini, and J. Wang, Phys. Rev. A 66, 013807 (2002).

${ }^{17}$ M. O. Scully and M. S. Zubairy, Quantum Optics (Cambridge University Press, Cambridge, 1997).

${ }^{18}$ D. T. Pegg, S. M. Barnett, and J. Jeffers, Phys. Rev. A 66, 022106 (2002).

${ }^{19}$ R. Jozsa, J. Mod. Opt. 41, 2315 (1994).

${ }^{20}$ N. A. Peters, T.-C. Wei, and P. G. Kwiat, quant-ph/0407172 (unpublished).

${ }^{21}$ M. A. Nielsen and I. L. Cheung, Quantum Computation and Information (Cambridge University Press, Cambridge, 2000).
${ }^{22}$ R. J. Richards, An Introduction to Dynamics and Control (Longman Press, 1979).

${ }^{23}$ H. J. Carmichael, An Open System Approach to Quantum Optics, Lecture Notes in Physics Vol. 18 (Springer, Berlin, 1993).

${ }^{24}$ H. M. Wiseman, Quantum Semiclassic. Opt. 8, 205 (1996).

${ }^{25}$ H. M. Wiseman and G. E. Toombes, Phys. Rev. A 60, 2474 (1999).

${ }^{26}$ S. G. Schirmer, T. Zhang, and J. V. Leahy, J. Phys. A 37, 1389 (2004); G. Kimura and A. Kossakowski, quant-ph/0408014 (unpublished). 